\title{
Role of dynamic exchange coupling in magnetic relaxations of metallic multilayer films (invited)
}

\author{
B. Heinrich, G. Woltersdorf, ${ }^{\text {a) }}$ and R. Urban \\ Simon Fraser University, 8888 University Drive, Burnaby, British Columbia, V5A 1S6, Canada \\ E. Simanek \\ 6255 Charing Lane, Cambria, California 93428
}

(Presented on 14 November 2002)

\begin{abstract}
The relaxation processes were investigated by ferromagnetic resonance (FMR) using magnetic single, $\mathrm{Au} / \mathrm{Fe} / \mathrm{GaAs}(001)$, and double layer, $\mathrm{Au} / \mathrm{Fe} / \mathrm{Au} / \mathrm{Fe} / \mathrm{GaAs}(001)$, structures prepared by molecular beam epitaxy. These structures provided an excellent opportunity to investigate nonlocal damping which is caused by spin transport across a nonmagnetic spacer. In the double layer structures thin Fe layers $F 1$ were separated from a second thick Fe layer $F 2$ by a $\mathrm{Au}(001)$, normal metal spacer. The interface magnetic anisotropies separated the FMR fields of $F 1$ and $F 2$ by a big margin which allowed us to investigate FMR in $F 1$ while $F 2$ had a negligible angle of precession. The main result is that the ultrathin Fe films in magnetic double layers acquire a nonlocal interface Gilbert damping. Several mechanisms have been put forward to explain the nonlocal damping. A brief review of each mechanism will be presented. They will be compared with the experimental results allowing one to critically assess their applicability and strength. It will be shown that the precessing layers act as spin pumps and spin sinks. This concept was tested by investigating the FMR linewidth around an accidental crossover of the resonance fields for the layers $F 1$ and $F 2$.

(C) 2003 American Institute of Physics. [DOI: 10.1063/1.1543852]
\end{abstract}

\section{INTRODUCTION}

The small lateral dimensions of spintronics devices and high density memory bits require the use of magnetic metallic ultrathin film structures where the magnetic moments across the film thickness are locked together by the intra layer exchange coupling. Spintronics and high density magnetic recording employ fast magnetization reversal processes. It is currently of considerable interest to acquire a thorough understanding of the spin dynamics and magnetic relaxation processes in the nano-second time regime. The spin dynamics in the classical limit can be described by the Landau-Lifshitz-Gilbert equation of motion

$$
\frac{1}{\gamma} \frac{\partial \mathbf{M}}{\partial t}=-\left[\mathbf{M} \times \mathbf{H}_{\mathrm{eff}}\right]+\frac{G}{\gamma^{2} M_{s}^{2}}\left[\mathbf{M} \times \frac{\partial \mathbf{M}}{\partial t}\right],
$$

where $\gamma$ is the absolute value of the electron gyromagnetic ratio, $M_{s}$ is the saturation magnetization and $G$ is the Gilbert damping parameter. The effective field $\mathbf{H}_{\text {eff }}$ is given by the derivatives of the Gibbs energy, $U$, with respect to the components $\left(M_{x}, M_{y}, M_{z}\right)$ of the magnetization vector $\mathbf{M}(t)$, see. ${ }^{1}$ The second term in Eq. (1) represents the well known Gilbert damping torque. The purpose of this article is to review the basic concepts of magnetic relaxations with emphasis on metallic multilayers.

\footnotetext{
a) Author to whom correspondence should be addressed; electronic mail: gwolters@sfu.ca
}

\section{NONLOCAL DAMPING, EXPERIMENT}

The role of interface damping was investigated in high quality crystalline $\mathrm{Au} / \mathrm{Fe} / \mathrm{Au} / \mathrm{Fe}(001)$ structures grown on GaAs(001) substrates. ${ }^{2,26,27}$ The in-plane ferromagnetic resonance (FMR) experiments were carried out using 10, 24, 36, and $72 \mathrm{GHz}$ systems. ${ }^{3}$

Single Fe ultrathin films with thicknesses of $8,11,16$, 21, and 31 monolayers (MLs) were grown directly on GaAs(001). They were covered by a $20 \mathrm{ML}$ thick $\mathrm{Au}(001)$ cap layer for protection in ambient conditions. FMR measurements were used to determine the in-plane four-fold and uniaxial magnetic anisotropies, $K_{1}$ and $K_{u}$, and the effective demagnetizing field perpendicular to the film surface, $4 \pi M_{\text {eff }}$, as a function of the film thickness $d .^{3}$ The magnetic anisotropies were well described by the bulk and interface magnetic properties, respectively. ${ }^{2}$ The reproducible magnetic anisotropies and small FMR linewidths provided an excellent opportunity for the investigation of nonlocal relaxation processes in magnetic multilayer films. The thin $\mathrm{Fe}$ films which were studied in the single layer structures were regrown as a part of magnetic double layer structures. The thin Fe film $(F 1)$ was separated from the second thick layer $(F 2)$ by a $\mathrm{Au}(001)$ spacer $(N)$ of a variable thickness between 12 and $100 \mathrm{ML}$. The magnetic double layers were covered by a $20 \mathrm{ML} \mathrm{Au}(001)$ capping layer. The thickness of the $\mathrm{Au}$ spacer layer was always smaller than the electron mean free path $(38 \mathrm{~nm}),{ }^{4}$ and hence allowed ballistic spin transfer between the magnetic layers.

The interface magnetic anisotropies separated the FMR fields of $F 1$ and $F 2$ by a big margin $(\sim 1$ kOe, see Fig. 1$)$ allowing us to carry out FMR measurements in $F 1$ with $F 2$ 


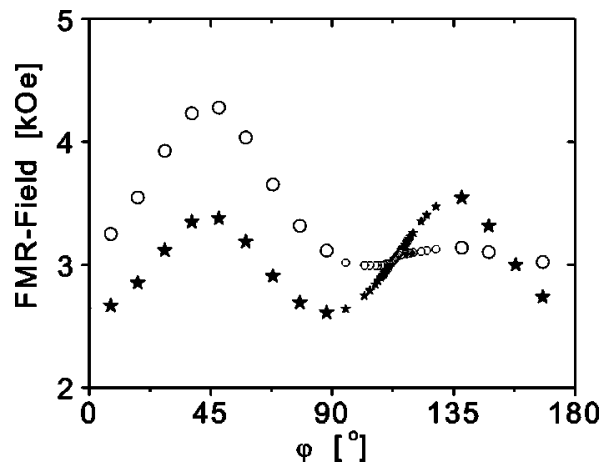

FIG. 1. The resonance fields at $24 \mathrm{GHz}$ in the layer $F 1[16 \mathrm{Fe}$, shown by $(\bigcirc)]$ and layer $F 2[40 \mathrm{Fe},(\star)]$ in $20 \mathrm{Au} / 40 \mathrm{Fe} / 16 \mathrm{Au} / 16 \mathrm{Fe} / \mathrm{GaAs}(001)$ (the integers represent the number of atomic layers) as a function of the angle $\varphi$ between the applied field and the in-plane [100] crystallographic direction. A large in-plane uniaxial anisotropy field $(0.5 \mathrm{kOe}$ with the hard axis along [1]10]) in $F 1$, is caused by dangling bonds of the $\mathrm{GaAs}(001)$ substrate, leads to an accidental crossover at $\varphi=115^{\circ}$ and $150^{\circ}$. Notice that the resonance fields get locked together by the spin pumping effect at the accidental crossover. Away from the crossover the resonance fields are separated by as much as ten FMR linewidths.

possessing a small angle of precession compared to that in $F 1$ and vice versa. The thin Fe film in the single and double layer structures had the same FMR field showing that the static interlayer exchange coupling ${ }^{1}$ through the Au spacer was negligible.

The main results are as follows: (a) The FMR linewidth in the thin films $F 1$ always increased in the presence of a thick layer $F 2$ and vice versa; (b) The additional FMR linewidth, $\Delta H_{\text {add }}$, followed an inverse dependence on the thin film thickness $d_{1} ;{ }^{2}$ and (c) the additional FMR linewidth $\Delta H_{\text {add }}$ in both the parallel (H in-plane) and perpendicular ( $\mathrm{H}$ perpendicular to the plane) FMR configuration was linearly dependent on the microwave frequency with no constant offset. The additional Gilbert damping for the $16 \mathrm{ML}$ thick film was found to be only weakly dependent on the crystallographic direction, with the average value $G_{\text {add }}=1.2 \times 10^{8}$ $\mathrm{s}^{-1}$. Its strength is comparable to the intrinsic Gilbert damping in the single Fe film, $1.4 \times 10^{8} \mathrm{~s}^{-1}$.

\section{THEORETICAL MODELS OF NONLOCAL DAMPING}

Berger ${ }^{5}$ evaluated the role of the s-d exchange interaction in magnetic double layers by allowing the magnetic moment of one layer $(F 1)$ to precess around the equilibrium direction while the other layer $(F 2)$ was assumed to be stationary, see the graphical representation in Fig. 2(a). Itinerant electrons entering the layer $F 1$ through a sharp interface cannot immediately accommodate the direction of the precessing magnetization. Berger showed that this leads to an additional exchange torque which is directed towards the magnetic equilibrium axis, and represents an additional relaxation term. This relaxation torque is confined to a region near the $F 1 / N$ interface whose thickness is given by the transverse spin relaxation coherence length $L_{0}=\pi /\left(k_{\uparrow}\right.$ $-k_{\downarrow}$ ), where $k_{\uparrow}$ and $k_{\downarrow}$ are the majority and minority Fermi $k$ wave vectors in $F 1$. $L_{0}$ is expected to be less than $1 \mathrm{~nm}$.
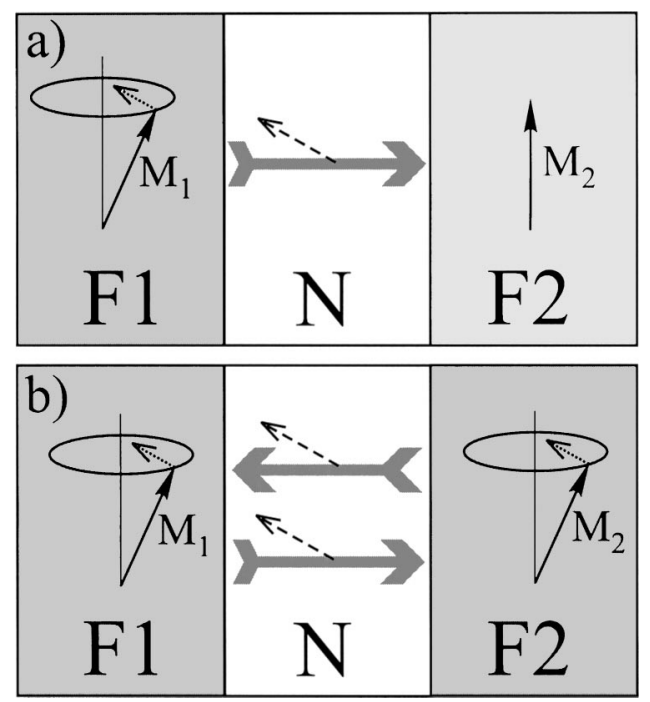

FIG. 2. An image representing the dynamic coupling between two magnetic layers which are separated by a nonmagnetic spacer $N$. (a) represents two magnetic layers with different FMR fields. $F 1$ is at resonance, and $F 2$ is nearly stationary. A large gray arrow in the normal spacer describes the direction of the spin current. The dashed lines represent the instantaneous direction of the spin momentum. For small angle of precession they are nearly parallel to the transverse rf magnetization component shown in short solid arrows. $F 1$ acts as a spin pump, $F 2$ acts as a spin sink. (b) represents a situation when $F 1$ and $F 2$ resonate at the same field. Both layers act as spin pumps and spin sinks. In this case the net spin momentum transfer across each interface is zero. No additional damping is present.

The resulting relaxation torque in a magnetic double layer structure contributes to an additional interface FMR linewidth $\Delta H_{\text {add }}$, such that

$$
\Delta H_{\text {add }} \sim(\Delta \mu+\hbar \omega),
$$

where $\Delta \mu=\Delta \mu_{\uparrow}-\Delta \mu_{\downarrow}$ is the difference in the spin up and spin down Fermi level shifts, and $\omega$ is the microwave angular frequency. $\Delta \mu$ is negligible for small angle precession, but can be brought in with + and - sign by a dc current which is oriented perpendicular to interfaces. ${ }^{6}$ The frequency dependent term in Eq. (2) was obtained using the full dynamic treatment of the $s-d$ exchange interaction, and it is always positive.

Berger's expression for the FMR linewidth, Eq. (2), was derived for a circular precession. One has to ask, what can be expected for the parallel FMR configuration where the demagnetizing effect leads to a strong ellipticity in precessional motion. Berger ${ }^{7}$ included the contribution in Eq. (2) to the nonlocal damping by using Slonczewski's spin transport torque. ${ }^{6}$ In this case the effective damping field for $F 1$ can be written as

$$
\operatorname{coef}(\Delta \mu+\hbar \omega) \mathbf{c} \times \frac{\mathbf{M}_{1}}{M_{s}} .
$$

where $\mathbf{c}$ is the direction of the magnetization in the stationary layer $F 2$, and coef is a common prefactor. The vector product $\mathbf{c} \times \mathbf{M}_{1}$ in the effective field results in Bloch-Blombergen damping with the relaxation rate parameter proportional to the microwave frequency. In the perpendicular configuration Eq. (3) results in the FMR linewidth which is strictly proportional to the microwave frequency (Gilbert-like), but for the 
parallel configuration the FMR linewidth is proportional to $\sim(\omega / \gamma)^{2} /(B+H)$. In the parallel configuration the FMR linewidth is dependent on the ellipticity of precession.

Tserkovnyak et $a .^{8}{ }^{8}$ showed that the interface damping can be generated by a spin current from a ferromagnet $(F)$ into the adjacent normal metal (NM) reservoirs. The spin current is generated by a precessing magnetic moment. A precessing magnetization at the $F / \mathrm{NM}$ interface acts as a "peristaltic spin-pump." The direction of the spin current is perpendicular to the $F / \mathrm{NM}$ interface and points away from the interface towards the NM layer. The spin momentum which is carried away by the spin current is

$$
\mathbf{j}_{\text {spin }}=\frac{\hbar}{4 \pi} A_{r} \mathbf{m} \times \frac{d \mathbf{m}}{d t},
$$

where $\mathbf{m}$ is the $a$ unit vector in the direction of $\mathbf{M}$. The spin current can result (see below) in magnetic damping. $A_{r}$ for $F$ films thicker than $L_{0}$ is given by the scattering matrix elements

$$
A_{r}=\frac{1}{2} \sum_{m, n}\left|r_{m n}^{\uparrow}-r_{m n}^{\downarrow}\right|^{2},
$$

where $r_{m n}^{\uparrow \downarrow}$ are the reflection parameters at the NM/F interface for the spin up and down electrons. The sum in $A_{r}$ is close to the number of the transverse channels in NM. ${ }^{9}$ The sum is given by

$$
\frac{A_{r}}{S}=\frac{k_{F}^{2}}{4 \pi}=0.85 n^{2 / 3},
$$

where $S$ is the area of the interface, $k_{F}$ is the Fermi wave vector, and $n$ is the density of electrons per spin in NM. ${ }^{9}$ Brataas et al. ${ }^{9,10}$ showed that $A_{r}$ can be evaluated from the interface mixing conductance $G_{\uparrow \downarrow} \cdot{ }^{11} A_{r}=\left(h / e^{2}\right) G_{\uparrow \downarrow}$ $=S g_{\uparrow \downarrow}$, where $g_{\uparrow \downarrow}$ represents "dimensionless interface mixing conductivity."

Now another important point has to be answered: "How is the generated spin current dissipated in the normal metal spacer $N$ ?" This answer can be found in Refs. 10 and 12. These authors have shown that the transverse component of the spin current in $N$ is entirely absorbed at the N/F2 interface [see Fig. 2(a)]. For small precessional angles the spin current is almost entirely transverse. This means that the $N / F 2$ interface acts as an ideal spin sink, and provides an effective spin brake for the precessing magnetic moment in $F 1$. The spin momentum $\mathbf{j}_{\text {spin }}$ in the spin current has the form of Gilbert damping in $F 1$. The Gilbert damping is given by the conservation of the total spin momentum

$$
\mathbf{j}_{\text {spin }}-\frac{1}{\gamma} \frac{\partial \mathbf{M}_{\text {tot }}}{\partial t}=0,
$$

where $\mathbf{M}_{\text {tot }}$ is the total magnetic moment in $F 1$. After simple algebraical steps one obtains an expression for the dimensionless spin pump contribution $\alpha_{\mathrm{sp}}$ to the damping

$$
\alpha_{\mathrm{sp}}=\frac{G_{\mathrm{sp}}}{\gamma M_{s}}=g \mu_{B} \frac{g_{\uparrow \downarrow}}{4 \pi M_{s}} \frac{1}{d_{1}},
$$

where $d_{1}$ is the thickness of $F 1, g_{\uparrow \downarrow}$ is the dimensionless mixing conductivity, and $G_{\mathrm{sp}}$ is the spin pump Gilbert pa- rameter. $g$ is the electron $g$ factor. The inverse dependence of $\alpha_{\text {sp }}$ on the film thickness clearly testifies to its interfacial origin. The layers $F 1$ and $F 2$ act as mutual spin pumps and spin sinks. For small precessional angles the equation of motion for $F 1$ can be written as

$$
\begin{aligned}
\frac{1}{\gamma} \frac{\partial \mathbf{M}_{1}}{\partial t}= & -\left[\mathbf{M}_{1} \times \mathbf{H}_{\mathrm{eff}, 1}\right]+\frac{G_{1}}{\gamma^{2} M_{s}^{2}}\left[\mathbf{M}_{1} \times \frac{\partial \mathbf{M}_{1}}{\partial t}\right] \\
& +\frac{\hbar}{4 \pi d_{1}} g_{\uparrow \downarrow, 1} \mathbf{m}_{1} \times \frac{\partial \mathbf{m}_{1}}{\partial t}-\frac{\hbar}{4 \pi d_{1}} g_{\uparrow \downarrow, 2} \mathbf{m}_{2} \frac{\partial \mathbf{m}_{2}}{\partial t},
\end{aligned}
$$

where $\mathbf{M}_{1}$ is the magnetization vector of $F 1, \mathbf{m}_{1,2}$ are the unit vectors along $\mathbf{M}_{1,2}$, and $d_{1}$ is the thicknesses of $F 1$. The exchange of spin currents is a symmetric concept and the equation of motion for the layer $F 2$ is obtained by interchanging the indices $1 \rightleftarrows 2$.

The spin pump model is a rather exotic theory to those who are used to magnetic studies. One would expect that there is a direct connection to a more common concept which is applicable to magnetic multilayers. The obvious choice is interlayer exchange coupling. The interlayer exchange interaction has been so far treated only in the static limit. ${ }^{13}$ One would expect that its dynamic part could create magnetic damping. A ferromagnetic sheet surrounded by a NM reservoir can be investigated by using a contact exchange interaction between the ferromagnetic spins and the electrons in NM. A similar model was used by Yafet $^{14}$ for calculating the static interlayer coupling. One can expand the linear response Kubo theory ${ }^{15}$ for slow precessional motion using a linear approximation for a retarded magnetic moment

$$
\mathbf{S}(t-\tau) \cong \mathbf{S}(t)-\tau \frac{\partial \mathbf{S}(t)}{\partial t},
$$

where $\mathbf{S}(t)$ is the spin moment of the magnetic sheet at the instantaneous time $\mathrm{t}$ and $\tau$ is the time delay of the retarded response. The induced moment in NM at the $F / \mathrm{NM}$ interface results in an effective damping field which is proportional to the imaginary part of the rf transverse susceptibility of NM and the time derivative of the magnetic moment

$$
\mathbf{H}_{\mathrm{damp}}^{\text {sd }} \sim\left[\frac{\partial}{\partial \omega} \int_{-\infty}^{\infty} \frac{d q}{2 \pi} \operatorname{Im} \chi(q, \omega)\right]_{\omega \rightarrow 0} \frac{d \mathbf{M}(t)}{d t} .
$$

This damping term satisfies again the Gilbert phenomenology. By using the same interaction potential it is shown ${ }^{16,28}$ that the Gilbert damping from the dynamic interlayer exchange coupling, $G_{s-d}$, is similar to that using the spinpumping theory ${ }^{8}$ combined with a perfect spin sink. This leads to an important conclusion: The spin pumping theory is equivalent to the dynamic response of the interlayer exchange coupling. The rf susceptibility in Eq. (11) allows one to account for electron-electron correlation effects in the normal metal. It has been shown ${ }^{16,28}$ that the Gilbert damping is enhanced by the square of the Stoner factor $S_{E}=[1$ $\left.-U N\left(E_{F}\right)\right]^{-1}$,

$$
G_{s-d}^{\mathrm{enh}}=G_{s-d} S_{E}^{2},
$$


where $U$ is the screened interatomic Coulomb interaction and $N\left(E_{F}\right)$ the electron density of states, per atom, at the Fermi level in NM.

It is worthwhile to realize that the $s-d$ exchange relaxation mechanism also applies to bulk ferromagnets, and was evaluated by Heinrich et al. ${ }^{17,18}$ The Gilbert damping in this case is given by

$$
G_{s-d}^{\text {bulk }}=\frac{\chi_{P}}{\tau_{\text {sf }}},
$$

where $\chi_{P}$ is the Pauli susceptibility and $\tau_{\mathrm{sf}}$ is the spin flip relaxation time of itinerant electrons in the ferromagnet. It should be noted that $1 / \tau_{\mathrm{sf}}$ in metals is proportional to the square of the spin orbit interaction. ${ }^{17,18}$ Using $\chi_{P}$ from Kriesman and Callen ${ }^{19}$ and $\tau_{\text {sf }}$ from the spin diffusion length in current perpendicular to plane giant magnetoresonance measurements one obtains for the bulk Gilbert damping $G=5$ $\times 10^{6}$ and $1 \times 10^{8} \mathrm{~s}^{-1}$ for Co and permalloy (Py), respectively, see the details in Ref. 18. This contribution is small in Co but it explains the intrinsic damping in $\mathrm{Py} . \mathrm{Fe}$ is expected to behave like Co. The spin pumping mechanism is very effective for ultrathin films, but is negligible in bulk materials because its strength is inversely proportional to the thickness. Notice that the spin pumping mechanism does not have an explicit temperature dependence, while the bulk Gilbert damping [see Eq. (13)], scales with $1 / \tau_{\mathrm{sf}}$ which is proportional to resistivity. One expects that there has to be an additional mechanism which depends explicitly on $\tau_{\mathrm{sf}}$. The origin of the interlayer exchange coupling lies in the itinerant nature of the electron carriers. It can be explained by using a spin dependent interface potential. ${ }^{20}$ The effective field that acts on the layer $F 1$ is given by differentiating the density of the interlayer exchange energy $E^{\text {int }}$ with respect to $\mathbf{M}_{1}$

$$
\mathbf{H}_{\mathrm{damp}}^{\mathrm{int}}=-\frac{\partial E^{\mathrm{int}}}{\partial \mathbf{M}_{1}}=-\frac{1}{\Omega} \sum_{k \sigma} n_{k, \sigma} \frac{\partial \boldsymbol{\epsilon}_{k, \sigma}}{\partial \mathbf{M}_{1}},
$$

where $n_{k, \sigma}$ and $\epsilon_{k, \sigma}$ are the occupation number and energy of electrons for the state described by the wave vector $\mathbf{k}$ and the spin $\sigma$ participating in the interlayer exchange coupling. These electrons are mostly confined to the $N$ spacer. $\Omega$ $=S d_{1}$ is the volume of the magnetic layer $F 1$. The energy of electrons is dependent on the instantaneous orientation of the magnetic moments, and consequently the occupation number $n_{k, \sigma}$ of electronic states having energy $\epsilon_{k, \sigma}$ changes with time and this results in a "breathing Fermi surface." This concept was also used in Refs. 21 and 22. However, this redistribution cannot be achieved instantaneously. The time lag between the instantaneous exchange field and the induced moment in the spacer is described by the transverse spin relaxation time $\tau_{\mathrm{sf}}$. In the limit of slow precessional motion the instantaneous electron distribution can be approximated by

$$
n_{k, \sigma}(t)=n_{k, \sigma}\left[\mathbf{M}_{\mathbf{1}}(t)\right]-\tau_{\mathrm{sf}} \frac{\partial n_{k, \sigma}\left[\mathbf{M}_{\mathbf{1}}(t)\right]}{\partial t},
$$

where $n_{k, \sigma}\left[\mathbf{M}_{\mathbf{1}}(t)\right]$ is the static occupation number for the magnetic moment of the layer $F 1$ with the magnetization along $\mathbf{M}_{\mathbf{1}}(t)$. The first term in Eq. (15) provides the static

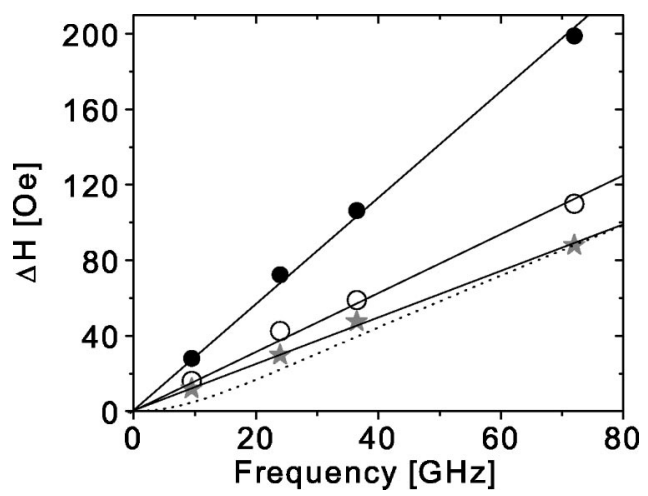

FIG. 3. The FMR linewidth for $16 \mathrm{Fe}(001)$ as a function of the microwave frequency using (O) $20 \mathrm{Au} / 16 \mathrm{Fe} / \mathrm{GaAs}(001)$ single and (O) $20 \mathrm{Au} / 40 \mathrm{Fe} /$ $40 \mathrm{Au} / 16 \mathrm{Fe} / \mathrm{GaAs}(001)$ double layer structure. ( $\star$ ) represent the additional part of the FMR linewidth $\Delta H_{\text {add }}$ in the double layer sample. The dotted line is a fit to the data obtained using Slonczewski's effective damping [see Eq. (3)].

interlayer exchange coupling field, and the second term provides damping. The effective damping field can be evaluated by using Eqs. (14) and (15):

$$
\mathbf{H}_{\mathrm{damp}}^{\mathrm{int}}=\tau_{\mathrm{sf}} \sum_{k, \sigma} \delta\left(\boldsymbol{\epsilon}_{k, \sigma}\left[\mathbf{M}_{\mathbf{1}}\right]-\boldsymbol{\epsilon}_{F}\right)\left(\frac{\partial \boldsymbol{\epsilon}_{k, \sigma}\left[\mathbf{M}_{1}\right]}{\partial \mathbf{M}_{\mathbf{1}}}\right)^{2} \frac{1}{d} \frac{\partial \mathbf{M}_{\mathbf{1}}}{\partial t},
$$

where the sum is carried out per unit area of $F 1$. This effective damping field is again proportional to the time derivative of the magnetic moment, and inversely proportional to the film thickness $d$; a clear indication of interface Gilbert damping. However in this case the damping field is proportional to the spin relaxation time $\tau_{\mathrm{sf}}$. Therefore this effect is explicitly dependent on the conductivity and represents a different contribution to the nonlocal damping compared to the spin pumping mechanism which is independent of $\tau_{\mathrm{sf}}$.

\section{DISCUSSION OF THE RESULTS}

Spin pumping and breathing Fermi surface theories predict a Gilbert damping having a strictly linear dependence of $\Delta H_{\text {add }}$ on the microwave frequency. Figure 3 shows that this is experimentally verified over a wide range of microwave frequencies. The dotted line represents the FMR linewidth calculated using Berger's effective field [see Eq. (3)]. Surprisingly even in this case the measured microwave frequency dependence of $\Delta H_{\text {add }}$ is essentially linear. The difference between the Gilbert damping and Berger's damping is only apparent in the negative zero frequency offset (obtained by extrapolating the dotted line to zero microwave frequency). The fit using the the dotted line is obviously poorer than that using the straight line for Gilbert damping. The spin pumping theory is clearly the mechanism of preference for the nonlocal damping. Its validity can be tested by comparing calculations using Eq. (9) with the experimental results. Figure 2 shows two extreme situations. In Fig. 2(a) the FMR fields in $F 1$ and $F 2$ are separated by a big margin. In Fig. 2(b) the FMR fields are the same. In (a) one expects the full contribution from the nonlocal damping. $\Delta H_{\text {add }}$ for $F 1$ and $F 2$ should scale with their respective $1 / d$ terms. In (b) the 


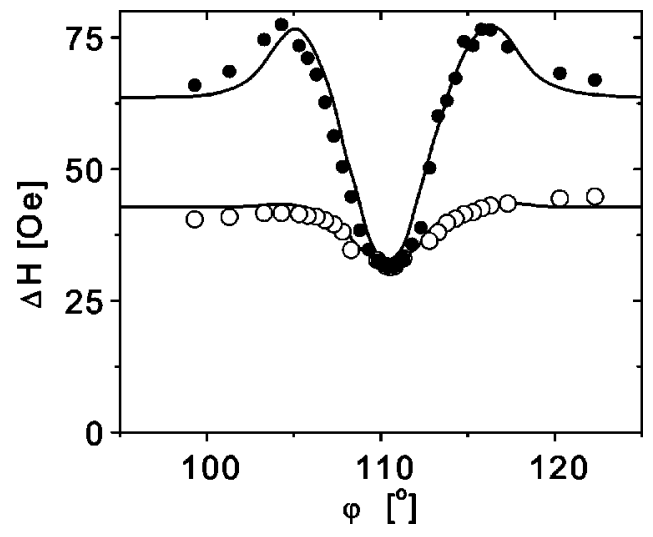

FIG. 4. The FMR linewidth at $24 \mathrm{GHz}$ as a function of the angle $\varphi$ around the crossover of the FMR fields for $20 \mathrm{Au} / 40 \mathrm{Fe} / 14 \mathrm{Au} / 16 \mathrm{Fe} / \mathrm{GaAs}(001)$. The measured and calculated FMR signals were analyzed using two Lorenzian lineshapes. The Lorenzian peaks were characterized by their amplitudes, resonance fields and linewidths. The solid lines were obtained from calculations using Eq. (9). The position of the FMR peaks is shown in Fig. 3. ( ) correspond to $F 1(16 \mathrm{Fe})(\bigcirc)$ correspond to $F 2(40 \mathrm{ML})$. Note that the FMR linewidth for the thinner sample, $F 1$, first increases before it reaches its minimum value corresponding to its single $20 \mathrm{Au} / 16 \mathrm{Fe} / \mathrm{GaAs}(001)$ layer structure. Note also that the additional line broadening scales inversely with the film thickness.

situation is symmetric, the net spin momentum flow through both interfaces is zero, and no additional damping is expected. This behavior is well demonstrated in Fig. 4. The good agreement between theory and experiment clearly shows the validity of the spin pumping theory which is described by Eq. (9). The magnetic layers even in the absence of static interlayer exchange coupling are coupled by the dynamic part of interlayer exchange. The spin sink effect at the $N / F$ interface starts to be inefficient only when the $N$ metal spacer thickness becomes comparable to the spin diffusion length. The spin diffusion length in $\mathrm{Au}$ is of the order of $100 \mathrm{~nm}$. The static interlayer exchange coupling vanishes in our samples due to interface roughness on a length scale of a mere $10 \mathrm{ML}(2 \mathrm{~nm})$. One should point out that when the $N$ metal spacer thickness starts to be comparable to the spin diffusion length then the $N$ spacer on its own can act as an effective spin sink. ${ }^{23,24}$

The quantitative comparison with predictions of the spin pumping theory is very favorable. First principles electron band calculations ${ }^{11}$ resulted in $g_{\uparrow \downarrow} \approx 1.1 \times 10^{15} \mathrm{~cm}^{-2}$ for an alloyed $\mathrm{Cu} / \mathrm{Co}(111)$ interface. By scaling this value to $\mathrm{Au}$ using Eq. (6) one obtains $G_{\mathrm{sp}}=1.4 \times 10^{8} \mathrm{~s}^{-1}$ which is close to that measured by FMR. This is a surprising agreement considering the fact that calculations of the intrinsic damping in bulk metals have been carried out over the last three decades, and yet they have not been able to produce a comparable agreement with experiment. ${ }^{18}$

The breathing Fermi surface contribution to the Gilbert damping is proportional to the electron relaxation time $\tau_{\text {sf }}$ of the $N$ metal spacer [see Eq. (16)]. A test of the breathing Fermi surface contribution can be carried out by measuring the temperature dependence of the nonlocal damping. One expects proportionality with the sheet conductance $\left(\tau_{\mathrm{sf}}\right.$ $\sim \tau_{\text {orb }} \sim \sigma$ ) of the $N$ spacer. The temperature dependence of the additional FMR linewidth, shown in Fig. 5, clearly indi-

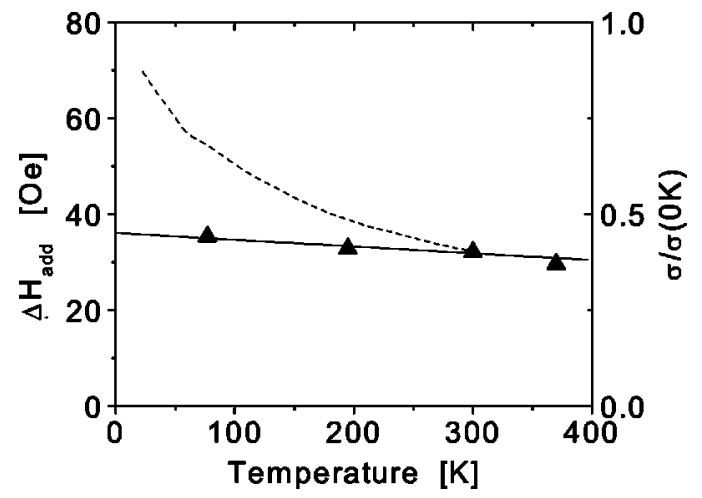

FIG. 5. The additional FMR linewidth, $\Delta H_{\text {add }}$, in $20 \mathrm{Au} / 14 \mathrm{Au} / 16 \mathrm{Fe} /$ $\mathrm{GaAs}(001)$ shown in black triangles, as a function of temperature. The temperature dependence of the sheet conductivity, $\sigma$, is shown in the dashed line. Note that the temperature dependence of $\Delta H_{\text {add }}$ is very weak.

cates that the strength of the breathing Fermi surface contribution is very small in $\mathrm{Fe} / \mathrm{Au} / \mathrm{Fe}(001)$. In fact, the observed temperature dependence of $\Delta H_{\text {add }}$ is caused by the presence of spin dependent resistance in the Au spacer, which will be discussed in a separate article.

The dynamic exchange coupling theory [see Eq. (11)], allows an enhancement of the additional Gilbert damping by the Stoner enhancement factor [see Eq. (12)]. In fact, our recent results using $20 \mathrm{Au} / 4 \mathrm{Pd} /[\mathrm{Fe} / \mathrm{Pd}]_{5} / 14 \mathrm{Fe} / \mathrm{GaAs}(001)$ single and $20 \mathrm{Au} / 40 \mathrm{Fe} / 40 \mathrm{Au} / 4 \mathrm{Pd} /[\mathrm{Fe} / \mathrm{Pd}]_{5} / 14 \mathrm{Fe} / \mathrm{GaAs}(001)$ double layer samples (see Fig. 6), show some evidence for the Stoner enhancement factor. This structure incorporates a magnetic $[\mathrm{Fe} / \mathrm{Pd}]_{5}$ superlattice with five repetitions of a $[1 \mathrm{Fe} / 1 \mathrm{Pd}]$ unit cell. The $N$ metal spacer is $4 \mathrm{Pd} 40 \mathrm{Au}(001)$. Note that at $\varphi=135^{\circ}$ the FMR linewidth is decreased down to the value which was observed for the single layer structure $\mathrm{GaAs} / 14 \mathrm{Fe}[1 \mathrm{Pd} / 1 \mathrm{Fe}]_{5} / 4 \mathrm{Pd} / 20 \mathrm{Au}(001)$. At $\varphi=135^{\circ}$ the resonant fields in the $14 \mathrm{Fe}[1 \mathrm{Pd} / 1 \mathrm{Fe}]_{5}$ and $40 \mathrm{Fe}$ layers were almost identical, eliminating the nonlocal damping. The additional FMR linewidth along the cubic crystallographic axes $\left(\varphi=0^{\circ}\right.$ and $\left.90^{\circ}\right)$ was enhanced by as much as a factor of 3 (see Fig. 6). The value of the nonlocal damping is significantly bigger than that expected from the simple spin pump-

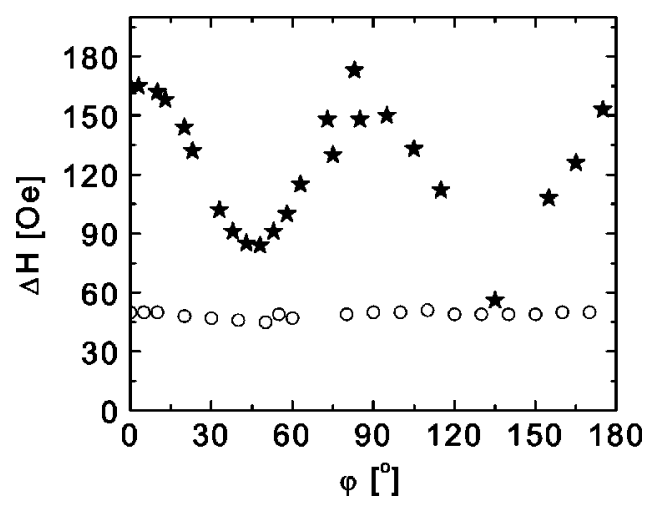

FIG. 6. The dependence of the FMR linewidth in $14 \mathrm{Fe}[1 \mathrm{Pd} / 1 \mathrm{Fe}]_{5}$ at $36 \mathrm{GHz}$ as a function of the angle $\varphi$. (O) symbols correspond to the single layer measurements using a GaAs$/ 14 \mathrm{Fe}[1 \mathrm{Pd} / 1 \mathrm{Fe}]_{5} / 4 \mathrm{Pd} / 20 \mathrm{Au}(001)$ structure, and ( $\star$ ) symbols correspond to the double layer measurements using a GaAs/ $14 \mathrm{Fe}[1 \mathrm{Pd} / 1 \mathrm{Fe}]_{5} / 4 \mathrm{Pd} / 40 \mathrm{Au} / 40 \mathrm{Fe} / 20 \mathrm{Au}(001)$ structure. 
ing mechanism. Metallic $\mathrm{Pd}$ is known to exhibit a strong Stoner enhancement in the dc susceptibility. These results clearly show that electron correlation effects in the $N$ metal spacer have to be seriously considered.

It is interesting to explore the role of spin pumping in a bilayer $5 \mathrm{Fe} / 12 \mathrm{Cu} / 10 \mathrm{Fe}(001)$ where the Fe layers are coupled by interlayer exchange energy. In this case one gets acoustic and optical precessional modes. ${ }^{1}$ Calculations were carried out at $36 \mathrm{GHz}$ using the spin pump and spin sink contributions as shown in Eq. (9). For a moderate antiferromagnetic exchange coupling $J=-0.2 \mathrm{ergs} / \mathrm{cm}^{2}$, the optical peak is broadened by 200 Oe while the acoustic peak is only broadened by 36 Oe. For antiferromagnetic exchange coupling the optical peak mostly arises from the $5 \mathrm{Fe}$ layer. For zero interlayer exchange coupling the spin pumping contribution to the FMR linewidth for the 5Fe layer is 150 Oe. This should be expected considering that the optical peak corresponds to an out of phase precession of the magnetic moments in the $5 \mathrm{Fe}$ and $10 \mathrm{Fe}$ layers, and therefore the spin momentum is more efficiently pumped. Experimentally, optical FMR peaks were always observed to be wider than the acoustic peaks. In a $5 \mathrm{Fe} / 12 \mathrm{Cu} / 10 \mathrm{Fe}$ sample grown on $\mathrm{Ag}(001)$ substrate the measured optical peak was broadened by 500 Oe. ${ }^{25}$ The above calculation indicates that approximately $50 \%$ of the broadening was due to spin pumping and $50 \%$ was caused by an inhomogeneous exchange coupling.

\section{CONCLUSIONS}

We have shown that nonlocal damping by the transfer of spin momentum can be realized in magnetic multilayer films. The effect is significant in ultrathin films. Theoretical models were presented for the nonlocal damping. It has been demonstrated that the nonlocal interface Gilbert damping in magnetic multilayers is well described by the concept of spin pumps and spin sinks. It has been shown that this effect is directly related to the dynamics of the interlayer exchange coupling. By proper engineering of multilayer structures one can create magnetic damping which significantly surpasses that in the bulk materials.

\section{ACKNOWLEDGMENTS}

The authors thank Y. Tserkovnyak, A. Brataas, G. E. W. Bauer, J. F. Cochran, and K. Myrtle for their assistance and valuable discussions during the course of this work. Financial support from the Natural Sciences and Engineering Research Council of Canada (NSERC) and Canadian Institute for Advanced Research (CIAR) is gratefully acknowledged. G.W. thanks the German Academic Exchange Service (DAAD) for generous financial support.

${ }^{1}$ B. Heinrich and J. F. Cochran, Adv. Phys. 42, 523 (1993).

${ }^{2}$ R. Urban, G. Woltersdorf, and B. Heinrich, Phys. Rev. Lett. 87, 217204 (2001).

${ }^{3}$ B. Heinrich and J. A. C. Bland, Ultrathin Magnetic Structures II (Springer, Berlin, 1994), Radio Frequency Techniques sections.

${ }^{4}$ A. Enders, T. Monchesky, K. Myrtle, R. Urban, B. Heinrich, J. Kirschner, X.-G. Zhang, and W. Butler, J. Appl. Phys. 89, 7110 (2001).

${ }^{5}$ L. Berger, Phys. Rev. B 54, 9353 (1996).

${ }^{6}$ J. C. Slonczewski, J. Magn. Magn. Mater. 159, 1 (1996).

${ }^{7}$ L. Berger, J. Appl. Phys. 91, 6795 (2002).

${ }^{8}$ Y. Tserkovnyak, A. Brataas, and G. Bauer, Phys. Rev. Lett. 88, 117601 (2002).

${ }^{9}$ A. Brataas, Y. Tserkovnyak, G. Bauer, and B. Halperin, Phys. Rev. B 66, 060404 (2002).

${ }^{10}$ A. Brataas, Y. Nazarov, and G. Bauer, Eur. Phys. J. E 22, 99 (2001).

${ }^{11}$ K. Xia, P. Kelly, G. Bauer, A. Brataas, and I. Turek, Phys. Rev. B 65, 220401 (2002).

${ }^{12}$ M. Stiles and A. Zangwill, J. Appl. Phys. 91, 6812 (2002).

${ }^{13}$ J. Slonczewski, J. Magn. Magn. Mater. 126, 374 (1993).

${ }^{14}$ Y. Yafet, Phys. Rev. B 36, 3948 (1987).

${ }^{15}$ S. Doniach and E. Sondheimer, Green's Functions for Solid State Physics (Benjamin, New York, 1974).

${ }^{16}$ E. Simanek and B. Heinrich, ArXivcond-mat/0207471 2002.

${ }^{17}$ B. Heinrich, D. Fraitová, and V. Kamberksý, Phys. Status Solidi 23, 501 (1967).

${ }^{18}$ B. Heinrich and J. A. C. Bland, Ultrathin Magnetic Structures III (Springer, Berlin, 2003), chap. B. Heinrich on Spin Relaxations in Magnetic Metallic Layers and Multilayers.

${ }^{19}$ C. Kriesman and H. Callen, Phys. Rev. 94, 837 (1954).

${ }^{20}$ P. Bruno, Phys. Rev. B 52, 411 (1995).

${ }^{21}$ V. Korenman and R. Prange, Phys. Rev. B 6, 2769 (1972)

${ }^{22}$ J. Kunes and V. Kamberský, Phys. Rev. B 65, 212411 (2002).

${ }^{23}$ R. Urban, G. Woltersdorf, and B. Heinrich, J. Appl. Phys. (to be published).

${ }^{24}$ S. Mizukami, Y. Ando, and T. Miyazaki, J. Magn. Magn. Mater. 226, 1640 (2001).

${ }^{25}$ B. Heinrich et al., Phys. Rev. Lett. 64, 673 (1990).

${ }^{26}$ B. Heinrich, R. Urban, and G. Woltersdorf, J. Appl. Phys. 91, 7523 (2002).

${ }^{27}$ B. Heinrich, R. Urban, and G. Woltersdorf, IEEE Trans. Magn. 38, 2496 (2002).

${ }^{28}$ B. Heinrich, G. Woltersdorf, R. Urban, and E. Simanek, J. Magn. Magn. Mater. (to be published). 